\title{
SMALLEST LECTIN-LIKE PEPTIDE IDENTIFIED FROM THE SKIN SECRETION OF AN ENDEMIC FROG, HYDROPHYLAX BAHUVISTARA
}

\author{
SHORT COMMUNICATION \\ Thundiparampil Vasanth Vineethrumar, Gopal Shyla and Sanil George* \\ Chemical and Environmental Biology group, Rajiv Gandhi Centre for Biotechnology, \\ Thiruvananthapuram-695014, Kerala, India
}

(Received: September 14, 2017; accepted: December 27, 2017)

\begin{abstract}
Lectins are sugar-binding proteins and considered as attractive candidates for drug delivery and targeting. Here, we report the identification of the smallest lectin-like peptide (odorranalectin HYba) from the skin secretion of Hydrophylax bahuvistara which is being the shortest lectin-like peptide identified so far from the frog skin secretion, with 15 amino acid residues. The peptide is the first report from an Indian frog and lacks antimicrobial activity but strongly agglutinate intact human erythrocytes. The sequences at the L-fucose recognizing region is conserved as in other lectins reported from frog skin secretion and could be exploited for specificity and drug targeting properties.
\end{abstract}

Keywords: Agglutination - amphibian - antimicrobial - Hydrophylax - lectin

Lectins are proteins that can bind to specific sugar residues and agglutinate cells. Typically they bind to cell surface glycoproteins and glycolipids [4]. Their binding is robust, rapid and is determined by specific sugar code. Lectins were first isolated from plants and thought to be only an agglutinating agent. As lectins were proved to be useful tools for the investigation of sugar moieties on the cell surface, especially on cancerous cells and as mediators for drug targeting, many lectins were reported from microorganisms and animals [4]. Currently, lectins are being increasingly investigated for drug targeting, which could prevent side effects to normal cells and enhance drug delivery to targeted cells [3]. The size of the lectin (more than $10 \mathrm{KDa}$ ) is one of the major problems which results in toxicity and immunogenicity and hence, shorter lectin-like molecules are at the forefront of the hunt [1]. Cationic host defense peptides (HDP) found in the skin secretion of frogs act as the first line immune defense protecting the animal from microbial infections and predators [7]. Lectin is one such a peptide that can agglutinate bacteria and fungus and is considered as a novel defense mechanism by amphibian immune system [2].

In the present study, a novel lectin-like peptide having homology to an odorranalectin family of peptides was identified from the skin secretion of Hydrophylax bahuvistara, an endemic frog species of Western Ghats, India. Skin secretion harvesting,

*Corresponding author; e-mail address: sgeorge@rgcb.res.in 
molecular cloning and primary structure elucidation of the peptides were done as per standard protocols [7]. Homology search of the peptide sequence was done using BLAST (NCBI), Peptide was chemically synthesized by Fmoc chemistry using CLEAR-amide resin, purity of the synthesized peptide were analyzed by MALDITOF-MS. ProtParm (http://expasy.org/tools/protparam.html) and Pepcalc (http:// pepcalc.com/) were used to compute physicochemical properties. Antimicrobial and hemagglutinating activity were determined as per standard procedures $[2,5]$.

cDNA sequence encoding the peptide with $91 \%$ identity to odorranalectin (17 residues; GenBank acc. no: ABW34702) reported from Odorrana grahami [5] was identified. Its open reading frame encodes a polypeptide composed of 59 amino acids, organized quite similarly to amphibian antimicrobial peptides. It consists of three regions, an $\mathrm{N}$-terminal signal peptide sequence ( 22 residues) followed by an acidic spacer (22 residues) and the C-terminal mature sequence (15 residues; Fig. 1A). There is a dibasic cleavage site (K43-R44) between the acidic spacer and mature peptide, which is believed to be cleaved by trypsin-like proteases. The peptide shares a similar signal peptide homology with the antimicrobial peptide lividin8 HYba isolated from $H$. bahuvistara [6]. This is the smallest peptide reported till date from the odorranalectin family, and we named it as odorranalectin HYba according to the nomenclature system for amphibian peptides [5] and submitted to GenBank (Acc. No: MF953660). The small size and single disulfide bridge may be an attracting feature of the peptide for the easy manipulation for developing as a drug targeting system [2]. This is the first lectin-like peptide identified from the skin secretion of an Indian frog and second from the Asian region. The synthesized mature peptide is cationic with +2 charges and molecular weight of $1640.94 \mathrm{Da}$ (Table 1A, Supplementary data $\mathrm{S} 1)$. It is weakly hydrophobic known from their negative GRAVY value $(-0.353)$ and is thought to have theoretical PI of 8.75.

Antimicrobial activity of odorranalectin HYba against Gram-positive and -negative bacteria was analyzed as they contain the signal and spacer similar to amphibian antimicrobial peptides (AMPs), but it did not show any activity against the tested Gram-positive and -negative bacteria in the peptide concentration range of $0.7-$

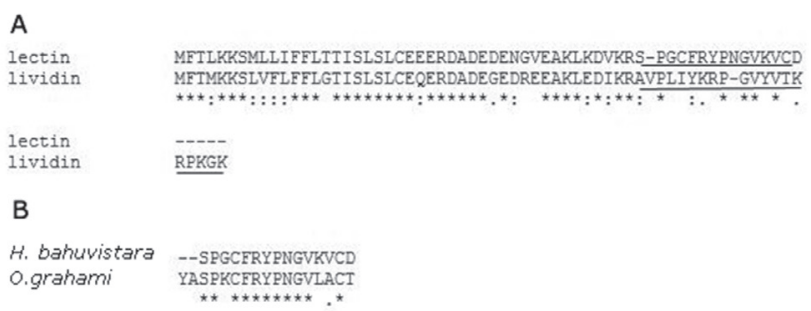

Fig. 1. (A) The precursor of odorranalectin HYba shares a similar signal peptide with the antimicrobial peptide lividin8 HYba isolated from H. bahuvistara (GenBank acc. no: AMD09936). Both of the mature peptides are preceded by a K-R endoproteolytic cleavage site. The mature peptide is underlined. *Indicates conserved amino acid residues. (B) Multiple Sequence Alignment of Odorranalectin from H. bahuvistara and O. grahami. *Indicates conserved amino acid residues. Sequences aligned using EMBL-MUSCLE 


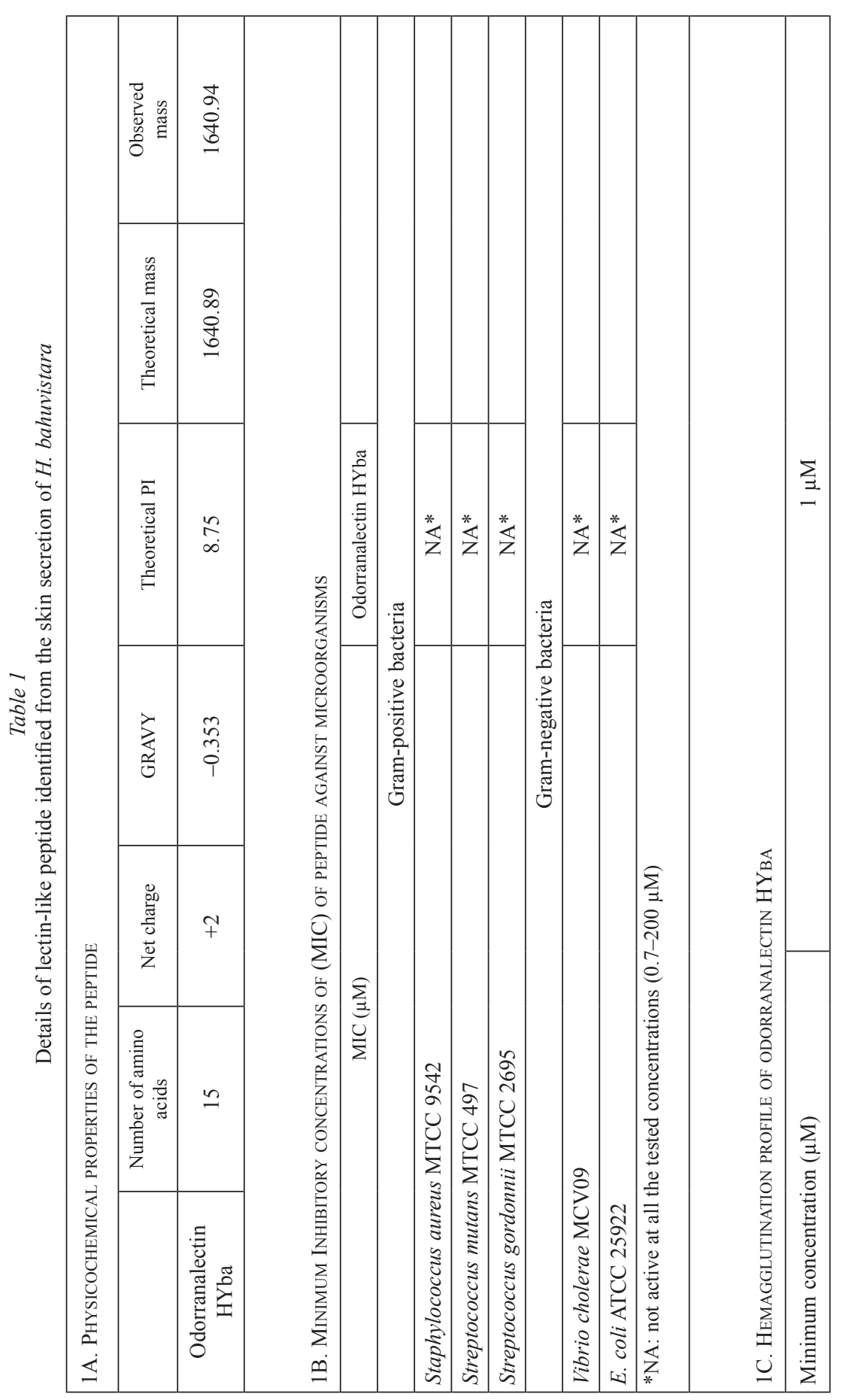

Acta Biologica Hungarica 69, 2018 
$200 \mu \mathrm{M}$. (Table 1B). The same observation was previously reported for odorranalectin from $O$. grahami [5]. Odorranalectin HYba strongly agglutinates intact human erythrocytes, the minimum peptide concentration for agglutination was found to be $1 \mu \mathrm{M}$ (Table $1 \mathrm{C}$ ), this is a signature activity shown by lectin family.

Comparing the primary structures of odorranolectin HYba and odorranolectin from $O$. grahami, it was found that the region in the peptide responsible for recognizing the specific carbohydrate residue is highly conserved (Fig. 1B). Thus it is possible to hypothesize that the peptide in the present study also could bind to L-fucose [2] and can be exploited further for drug delivery and targeting applications. The major advantage of this peptide would be its small size with all lectin functional domains conserved. The small size of the peptide could reduce the size of the nanocarriers, which would inturn enhance the delivery at the target site.

\section{ACKNOWLEDGEMENT}

KSCSTE, Kerala, is acknowledged for financial assistance and Kerala Forest Department for sampling permissions.

\section{REFERENCES}

1. Bies, C., Lehr, C. M., Woodley, J. F. (2004) Lectin-mediated drug targeting: history and applications. Drug Deliv. Rev. 56, 425-435.

2. Li, J., Wu, H., Hong, J., Xu, X., Yang, H. et al. (2008) Odorranalectin is a small peptide lectin with potential for drug delivery and targeting. PLOS ONE. 3, e2381.

3. Minko, T. (2004) Drug targeting to the colon with lectins and neoglycoconjugates. Adv. Drug Delivery Rev. 56, 491-509.

4. Sharon, N., Lis, H. (2004) History of lectins: from hemagglutinins to biological recognition molecules. Glycobiology 14, 53-62.

5. Thomas, P., Vineethkumar, T. V., Reshmy, V., Kumar, K. S., George, S. (2012) A mini review on the antimicrobial peptides isolated from the genus Hylarana (Amphibia: Anura) with a proposed nomenclature for amphibian skin peptides. Mol. Biol. Rep. 29, 6943-6947.

6. Vineethkumar, T. V., Shyla, G., George, S. (2016) First report of lividin and spinulosain peptides from the skin secretion of an Indian frog. Acta Biol. Hung. 67, 121-124.

7. Vineethkumar, T. V., Asha, R., Shyla, G., George, S. (2017) Identification and characterization of novel host defense peptides from the skin secretion of the fungoid frog, Hydrophylax bahuvistara (Anura: Ranidae). Chem. Biol. Drug Des. (in press). 